breeders during recent years will be read with much interest. A chapter on cultural matters concludes the introductory part of the work.

Mr. Millais is to be congratulated on the successful accomplishment of a very arduous task. He has brought together very nearly all the knowledge about rhododendrons that really matters - which is a great thing to have done. Handling his book, one could wish sometimes that it were not so large, but then we should have missed the fine coloured pictures by Miss Beatrice Parsons and Miss Winifred Walker. Other coloured plates by Miss Snelling are excellent, and besides all this the book is lavishly illustrated by reproductions from photographs of plants both wild and cultivated.

W. J. BEAN.

\section{The Origin of Solubility.}

Solubility. By Prof. Joel H. Hildebrand. (American Chemical Society Monograph Series.) Pp. 206. (New York: Chemical Catalog Co., Inc., I924.) 3 dollars.

$7 \mathrm{O}$ a reader who has not followed recent American 1 work on solubility, the contents of Prof. Hildebrand's book provide a pleasant surprise. Instead of a mere compendium of data, there is here a real attempt to develop a general theory of solubility, and of the even wider problems of miscibility in liquids, and of the properties of the resulting mixtures. The startingpoint of this general theory is not the familiar Law of Osmotic Pressure of van 't Hoff, but the more fundamental Law of Vapour Pressures of Raoult. Thus, in the case of certain mixtures, Raoult's law is valid over the whole range of concentrations from $\circ$ to Ioo per cent., whereas van 't Hoff's law is limited to dilute solutions and, even when modified, cannot be extended in a similar way. An ideal solution is therefore defined as one which obeys Raoult's law at all temperatures and all pressures. This depends on the internal forces remaining undisturbed on substituting molecules of solute for molecules of solvent. The author discusses what are the properties of the individual liquids, the equality of which is likely to lead to this result, and concludes that equality of "internal pressure" is the most important factor. Moreover, if this condition is realised at one temperature and pressure, it is likely to be realised over the whole range.

Deviations from Raoult's law are of two types. "Negative" deviations are accompanied by a contraction and evolution of heat on mixing, and may be accounted for, at least in some cases, by the formation of unstable compounds of solvent and solute. "Positive" deviations, accompanied by an expansion and absorption of heat on mixing, are less easily explained. In general they could be accounted for by the association of one of the pure liquids, so that dilution with an inert solute would give rise to dissociation; but, when this is regarded as the only possible explanation, it leads to the assumption of polymerised molecules of argon in solution in liquid nitrogen, and in general to the necessity for assuming an altogether unreasonable degree of molecular complexity in order to account for the larger deviations. Positive deviations from Raoult's law can also be " explained " by the existence of wide differences in " internal pressure," but this is in a sense little more than a paraphrase.

The author is, however, considerate enough to give a simpler (if less rigidly proved) explanation, by using the familiar classification of liquids into (i) "normal" liquids with low dielectric constants and small residual fields of force round the molecules, and (ii), "polar" liquids with high dielectric constants and large or unsymmetrical fields of force. The polar molecules, he says, have an abnormally great attraction for each other, thus producing greater cohesions, internal pressures, surface tensions and heats of vaporisation. They therefore tend "to squeeze out, as it were, nonpolar or slightly polar molecules from their midst"; and on account of this selective attraction of the polar molecules for each other, " the squeezing-out effect may be greater than it would be in the case of high internal pressure alone, unaccompanied by polarity." The result is a strong positive deviation from Raoult's law, with a corresponding influence on solubility. This classification is, of course, not a rigid one, but it agrees well with the familiar experience of all chemists that " if substances are divided into two groups, polar and non-polar respectively, those within either group are usually most soluble in the other substances within the same group and least soluble in those of the other group."

The summary given above of some of the points discussed by the author would probably give quite a wrong impression of the book, by suggesting that it is qualitative and non-mathematical in character. It may be taken as an unsolicited testimonial to the skill of the author that it should be possible to deal in this way with a book that is rigidly thermodynamical in its method of treatment. He has, indeed, written in such a way that a reader who is unskilled in thermodynamics and who "funks differentials" can nevertheless follow his argument with comfort. Such a reader is permitted to shut his eyes whilst traversing the thermodynamic tunnels, in the expectation that he will soon emerge, not merely into the daylight of an open cutting, but into broad stretches of open country. The book is therefore one that is well worth reading, and the author is to be congratulated on his success in presenting a difficult subject in so attractive a form.

T. M. L 